\title{
EVALUATION OF REFRACTIVE AND CORNEAL TOPOGRAPHIC CHANGES FOLLOWING UPPER EYELID BLEPHAROPLASTY
}

Amr Abdelazim Habib, Ibrahim YehiaAllam, Mai Abdallah Mohammed Moharam*

Department of Ophthalmology, Faculty of Medicine, Alexandria University, Resident at ministry of health*

\section{INTRODUCTION}

Blepharoplasty is a surgical procedure in which the eyelid skin, orbicularis oculi muscle, and orbital fat are excised, redraped, or sculpted to rejuvenate the esthetic look of the patient along with correction of any functional the esthetic
abnormality.

In upper eyelid blepharoplasty removal of excess skin and raising the eyelid may lead to redistribution of the pressure applied by the lids over the cornea and cause changes in corneal shape which could be proved with corneal topography. Also pressure of excess skin and prolapsed fat may cause alterations in normal corneal curvature. This could change corneal refraction or astigmatism and that may cause blurred vision.

\section{AM OF THE WORK}

Evaluation of refractive and corneal topographic changes that follows upper eyelid blepharoplasty.

\section{SUBJECTS AND METHODS}

\section{SUBJECTS}

Inclusioncriteria:

Patients with upper eyelid blepharochalasis without previous upper eyelid surgeries

Exclusioncriteria:

Exclusioncriteria: - dry

Previous coneal surgery, dry eye, uncontrolled diabetes milletus, kera

or keratectasia, corneal surface irregularities and lenticular pathologies.

METHODS

A prospective study in which10 patients(20 eyes) with age range from 35 to 81 years 2 of them were males(4eyes) and 8 females(16eyes) subjected to the following before undergoing upper eyelid blepharoplasty: Complete history taking including: Age, medical and surgical history.

Complete ophthalmic examination, Visual acuity testing, Cycloplgic refraction

Complete oplis

upper eyelid examition: marginal reflex distance(MRD), marginal crease

distance(MCD) and Pentacam (Oculyzer II, WaveLight, Germany) study of the cornea.
The acquired image was examined and the following measures were documented:

1- Thinnest location(in micrometer)

2-Kmax (in diopter)

3- Mean K (in diopter)

4-Corneal pattern configuration. As a result, four types of corneal topographic patterns were defined: symmetrical, asymmetrical, round and oval.

One month after operation all patients were subjected to: Visual acuity testing

Cycloplgic refraction and Pentacam (OculyzerII, Wave Light, Germany) study

of the cornea.

\section{RESULTS}

There were no statistical differences in refraction(astigmatism and spherical equivalent) between preoperative and one month postoperative data.

There were no statistical differences in corneal topography (thinnest location,

meanK, Kmax and corneal surface pattern) between preoperative and one

month postoperative data.

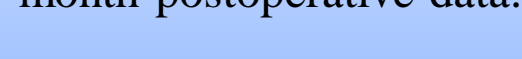

\begin{tabular}{|c|c|c|}
\hline Astigmatism & Pre & Post \\
\hline Min. - Max. & $-0.50--2.25$ & $-0.25--2.50$ \\
\hline Mean \pm SD. & $-0.90 \pm 0.51$ & $-0.93 \pm 0.60$ \\
\hline Median (IQR) & $-0.75(-1.0--0.50)$ & $-0.75(-1.0--0.50)$ \\
\hline P & \multicolumn{2}{|c|}{0.658} \\
\hline
\end{tabular}

Table 1:comparison between preoperative and postoperative astigmatism

\begin{tabular}{|l|c|c|}
\hline Thinnest location (um) & Preoperative & Postoperative \\
\hline Min. - Max. & $492.0-587.0$ & $488.0-594.0$ \\
\hline Mean \pm SD. & $542.3 \pm 31.41$ & $545.3 \pm 33.06$ \\
\hline Median (IQR) & $540.5(515.5-571.0)$ & $547.0(516.5-571.5)$ \\
\hline P & \multicolumn{2}{|c|}{0.111} \\
\hline
\end{tabular}

Table 2 :comparison between preoperative and postoperative thinnest location

\begin{tabular}{|c|c|c|}
\hline K max(D) & Pre & Post \\
\hline Min. - Max. & $42.70-46.50$ & $42.70-46.50$ \\
\hline Mean \pm SD. & $44.89 \pm 1.22$ & $44.90 \pm 1.15$ \\
\hline Median (IQR) & $45.25(43.85-45.80)$ & $45.35(43.90-45.75)$ \\
\hline P & \multicolumn{2}{|c|}{0.950} \\
\hline
\end{tabular}

Table 3 :comparison between preoperative and postoperative $\mathrm{K}$ max

\begin{tabular}{|c|c|c|}
\hline Km(D) & Preoperative & Postoperative \\
\hline Min. - Max. & $41.40-45.10$ & $41.50-45.20$ \\
\hline Mean \pm SD. & $43.70 \pm 1.17$ & $43.77 \pm 1.18$ \\
\hline Median (IQR) & $44.25(42.75-44.50)$ & $44.30(42.65-44.60)$ \\
\hline P & \multicolumn{2}{|c|}{0.120} \\
\hline
\end{tabular}

Table 4:comparison between preoperative and postoperative mean $K$.

\section{CONCLUSION}

- There were no statistically significant differences between preoperative and one month postoperative refraction (astigmatism and spherical equivalent) and corneal topography (thinnest location, mean $\mathrm{K}$,corneal surface pattern and $\mathrm{K}$ max).

- upper eyelid blepharoplasty has no effect on corneal topography and refraction.
2021@Alexandria Faculty of Medicine 